\title{
Histogram based Classification of Ultrasound Images of Placenta
}

\author{
G. Malathi \\ Affiliated to Anna University \\ Chennai
}

\author{
Dr. V. Shanthi \\ Affiliated to Anna University \\ Chennai
}

\begin{abstract}
In this paper, the authors have made an attempt to classify the placenta based on the intensity level of histogram of the ultrasound images of placenta. The medical images are usually low in resolution. Specialized tools are required to assist the medical experts in medical image diagnosis and for further treatment. The image histogram is used to classify the ultrasound images of placenta into normal and abnormal placenta using $\mathrm{k}$ nearest neighbor classifier. It is further used to analyze the complications of gestational diabetes mellitus on the growth of the placenta.
\end{abstract}

\section{Categories and Subject Descriptors}

Neural Computing - Image preprocessing - feature extraction feature selection - classification

\section{General Terms}

Algorithms, Neural Network, Image Processing

\section{Keywords}

placenta, $\mathrm{k}$ nearest neighbor, classification, intensity, histogram

\section{INTRODUCTION}

Gestational diabetes or gestational diabetes mellitus is a condition in which women without previously diagnosed diabetes exhibit high blood glucose levels during pregnancy. India is the Diabetic capital of the world. $80 \%$ of the diabetes in the world will be present in developing countries like India [1]. According to World Health Organization the number of diabetics through out the world was 171 millions in the year 2000. It was to become 336 millions by 2030 [2]. Chronic hypertension and diabetes mellitus may cause or aggravate such dysfunction thus causing placental abruption [3]. Placenta is the organ within the uterus by means of which the embryo is attached to the wall of the uterus. Its mail function is to provide the embryo with nourishment, eliminate its wastes and exchange respiratory gases [4].

Placenta is a curved structure and is too difficult to measures. If they had to measures the placental volume they would need a very expensive machine specialized training and more. The limitations in the current technology prevent monitoring the growth of the placenta. Fetal death, or intrauterine fetal demise affects 30,000 women every year in the US alone. [5].

Placental insufficiency is the failure of the placenta to supply nutrients to the fetus and remove toxic wastes. If placental insufficiency occurs for a long time during the pregnancy, it may lead to intrauterine growth retardation (IUGR). Between 3 to $7 \%$ of all pregn ancies are complicated by IUGR due to placental insufficiency and one of the reasons is diabetes [6]. Placenta size or shape alone may be sufficient to identify a subset of women at higher risk at the initial sound examinations [7]. In pregnancies complicated by placental dysfunction, there may be a reduction in the number of functional villi and /or small blood vessels with, as a result, increased impedance, reflected, mainly, by a decrease in end-diastolic velocity. When the resistance increases even more, there is no diastolic forward velocity (absent end-diastolic velocity). Further increase in the resistance causes reversed enddiastolic velocity, which is considered a late step in the cascade of events leading to intrauterine fetal demise. [8] Grannum et al. [11] reported the observation of subtle differences in the in vivo placenta using ultrasonic images in relation with different stages of pregnancy. As a result this study they proposed a 4-grade classification based on variations in placental appearance, which occur during gestation.

The assessment of placenta maturity is an important issue in prenatal diagnosis. The ability of a decision tree classifier to discriminate different textures with three sets of textural features was tested. The performance of the classifier using textural features corresponding to co-occurrence matrices, Law's operators, and neighborhood gray tone difference matrices was assessed and applied to the problem of the classification of ultrasonic images of the placenta corresponding to different grades. Ultrasonic images of the placenta according with the four classes proposed by Grannum were classified using decision trees built with C5.0 [9].

In order to improve the predictive accuracy of the classifier, the original number of feature set is reduced into smaller set using feature reduction techniques. Rough set based reduction algorithms such as Decision Relative Discernibility based reduction, Heuristic approach, Hu's algorithm, Quick Reduct and Variable Precision Rough Set are used to reduce the extracted features in [10]. In statistical approaches, texture statistics such as the moments of the gray-level histogram, or statistics based on gray-level co-occurrence matrix are computed to discriminate different textures [12]. In [13], the classification of scans of the placenta according with Grannum grading is attempted. Feature selection was used for determining the relevant textural features that were extracted from the scans. Three different sets of textural features, namely oc-occurrence matrices, Laws masks and neighborhood gray-tone difference matrices were used. The textural features were computed and weighed using the relief $F$ Algorithm. The strategy used for classification was the k-nearest neighbor algorithm using leave-one-out cross-validation. 


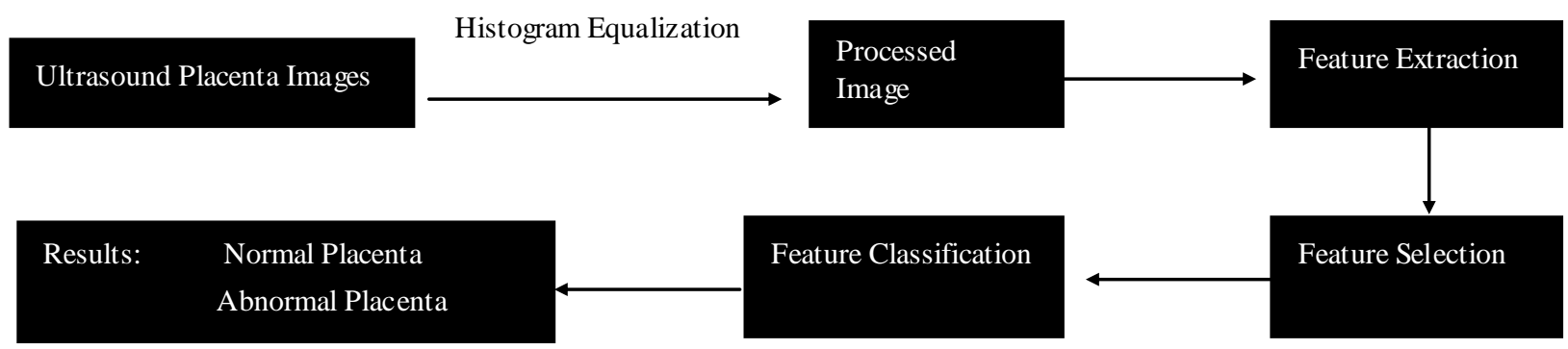

Figure 1 Ultrasound Placenta Images Classification Diagram

\section{FEATURE EXTRACTION}

Texture is one of the most important defining characteristics of an image. It is characterized by the spatial distribution of gray levels in a neighborhood. In order to capture the spatial dependence of gray-level value, which contributes to the perception of texture, a two dimensional dependence, and texture analysis matrix is considered. Since, texture shows its characteristics by both pixel and pixel values, there are many approaches used for texture classification [14]. The feature types can be divided into spectral, geometric and texture features

\subsection{Spectral Features}

When using spectral features the image is processed as a matrix of pixels. Each of these represents the brightness and color level that can be found at the corresponding locations in the image. The advantage of this type of feature is that they are independent of image size and coding transformations. [15]

\subsection{Shape Features}

It is very important for these features to be divided into two groups in the first are features that are invariant to translation, rotation, and scaling, while in the other group remain features that do not have these qualities. Features from the first group are normally simpler to extract and require simpler procedures. Procedures for extracting these features can also be divided into two groups. The first group represents boundary based procedures, which concentrate only on the outer boundary of the shape being extracted and use Fourier descriptors. The other group represents region based procedures which works with the whole shape as an object where invariant moments are used most often.

\subsection{Texture Features}

Texture features refer to visual patterns that have properties of homogeneity that do not result from the presence of only a single color or intensity. These features contain important information about the structural arrangement of surfaces and their relationship to the surrounding environment.[16]

\subsection{Color Histograms}

This technique is based on noting how much of a certain color is present in the current histogram. In order to use this technique is therefore necessary to segment the image before processing it. The advantage is that it is invariant to translation, rotation and scaling and therefore more reliable.

\subsection{Fourier Coefficients}

Fourier coefficients do not represent a new technique for feature extraction but instead rather present a simpler way of comparing features extracted from the image with those from database in order to identify objects in the image. [17]

\subsection{Wavelet Functions}

Wavelet $\Psi(\mathrm{t})$ is a function that satisfy the following condition:

$$
\int_{\mathrm{R}} \Psi \mathrm{dt}=0
$$

Wavelet bias functions are consisted of translating and dilating of a fixed wavelet function named mother wavelet. It can provide local spatial information and frequency information of a function or a signal, such local information for image classification. [18]

\section{IMAGE PRE-PROCESSING}

The ultrasonic images of placenta is obtained and submitted to a pre-processing for enhancement and improvement of the contrast using histogram equalization.

\section{STATISTICAL APPROACHES}

The key idea in the proposed technique is to classify the ultrasound images of placenta into interior pixels and boundary ones. The interior pixels stand for the interior parts of texture regions, then the segmentation can be achieved by applying adaptive region growing on the interior pixels. To implement this idea, the statistical properties of the intensity histogram are employed. The use of statistical moments based on histogram would clearly specify any distortions in the image. This would help the sonologist to classify the ultrasound images of placenta into normal and abnormal images and groups these images into four classes normal, placenta dysfunction, and placenta accreta and placenta previa.

\subsection{Statistical Moments}

A frequently used approach for texture analysis is based on statistical properties of intensity histogram. Once such measures is based on the statistical moments. Based on Haralick features in [19], mean, standard deviation, contrast, correlation and entropy were obtained from the ultrasound images of placenta. The following features were extracted from each of the gray-tone spatial-dependence matrices. $\mathrm{P}(\mathrm{i}, \mathrm{j})$ is the $(\mathrm{i}, \mathrm{j})$ th entry in a 
normalized gray-tone spatial dependence matrix. The gray level co-occurrence matrix is given by

$P_{d, i}(i, j)=P(i, j) / R$

\subsubsection{Mean}

It is the ith entry in the marginal-probability matrix obtained by summing the rows of $\mathrm{p}(\mathrm{i}, \mathrm{j})$,

$$
\begin{aligned}
& P_{y}(i)=\sum_{j=1}^{N_{j}} P(i, j) \\
& P_{y}(i)=\sum_{j=1}^{N_{j}} P(i, j)
\end{aligned}
$$

\subsubsection{Contrast}

The contrast feature is a difference moment of the $\mathrm{P}$ matrix and is a standard measurement of the amount of local variations presented in an image. The higher the value of contrast are, the sharper the structural variations in the image.

$f_{2}=\sum_{n=0}^{N_{n} 1} n^{2}\left\{\sum_{\substack{i=1 \\|i-j|=n}}^{N_{j i}} \sum_{j=1}^{N_{n}} P(i, j)\right\}$

Where,

$\mathbf{N}_{\mathrm{g}}$ is the number of distinct gray levels in the quantized image

\subsubsection{Correlation}

Correlation is the measure of similarity between two images in comparison

$f_{3}=\frac{\sum_{i} \sum_{j}(i j) \rho(i, j)-\mu_{i} \mu_{y}}{\sigma_{i} \sigma_{y}}$

Where

$\mu_{\mathrm{x}}$ and $\mu_{\mathrm{y}}$ is the mean

$\sigma_{\mathrm{x}}$ and $\sigma_{\mathrm{y}}$ is the standard deviation or the measure of contrast

\subsubsection{Entropy}

$f_{4}=-\sum_{i} \sum_{j} P(i, j) \log (P(i, j))$

The statistical moments obtained above were broadly classified into two groups as normal placenta and abnormal placenta. It is presented in the Table1. The abnormal placenta images were further classified into specific conditions of placenta during the gestational period.

\section{K-NEAREST NEIGHBOR CLASSIFICATION}

The training set includes the data for classification for each specific category of ultrasound placenta images.

For every new input, the textural features for mean, contrast, correlation and 5. entropy are obtained. It is presented in Table2. The new input is placed in the class that contains the most items which is close to the specific class of image.

The following are the steps of the algorithm

1. Given an input image $X$, determine its distance measure based on the computation of textural features.

2. Determine the $\mathrm{k}$ nearest neighbor in the training set which comprises of the haralick features.

3. Assign the image $\mathrm{X}$ to the classes of closest match.

Table 1. Haralick Features for Ultrasound Placenta Images(sample)

\begin{tabular}{|c|c|c|c|c|c|}
\hline Img & $\begin{array}{c}\text { Mean } \\
\left(\mathbf{e}^{4}\right)\end{array}$ & Contrast & Correlation & Entropy & Class \\
\hline Img1 & 1.635 & 1310473767 & $7.922339 \mathrm{e} 5$ & $8.944150 \mathrm{e} 4$ & $\mathrm{AN}$ \\
\hline Img2 & 1.832 & 1614852030 & $2.978678 \mathrm{e} 6$ & $1.047565 \mathrm{e} 5$ & $\mathrm{AN}$ \\
\hline Img3 & 1.454 & 1434646325 & $1.911394 \mathrm{e} 5$ & $9.609855 \mathrm{e} 4$ & $\mathrm{AN}$ \\
\hline Img4 & 1.455 & 1436691775 & $1.915322 \mathrm{e} 5$ & $9.670916 \mathrm{e} 4$ & $\mathrm{AN}$ \\
\hline Img5 & 1.222 & 1077321331 & $1.055089 \mathrm{e} 5$ & $7.347292 \mathrm{e} 4$ & $\mathrm{~N}$ \\
\hline Img6 & 1.832 & 1614852030 & $2.978678 \mathrm{e} 6$ & $1.047565 \mathrm{e} 5$ & $\mathrm{AN}$ \\
\hline Img7 & 1.854 & 1647605895 & $9.059511 \mathrm{e} 5$ & $7.575653 \mathrm{e} 4$ & $\mathrm{AN}$ \\
\hline Img8 & 1.749 & 1531849951 & $7.756140 \mathrm{e} 5$ & $9.824614 \mathrm{e} 4$ & $\mathrm{AN}$ \\
\hline Img9 & 1.263 & 1083142018 & $1.065980 \mathrm{e} 5$ & $7.394319 \mathrm{e} 4$ & $\mathrm{~N}$ \\
\hline Img10 & 1.280 & 1067278301 & $1.059341 \mathrm{e} 5$ & $7.367722 \mathrm{e} 4$ & $\mathrm{~N}$ \\
\hline
\end{tabular}

The abnormal image1 is classified as placenta abruption, image2 is classified as condition called placenta accreta, image 3 is classified as condition called GDM complicating pregnancy leading to placental dysfunction and image 5 classified as normal placenta. It is presented in Table2. 
Table 2. Classification of ultrasound placenta images based on Haralick features

\begin{tabular}{|c|c|c|c|c|c|}
\hline $\begin{array}{c}\text { Featur } \\
\text { es }\end{array}$ & Mean & Contrast & Correlation & Entropy & Class \\
\hline Image1 & $1.635 \mathrm{e} 4$ & 1310473767 & $7.922339 \mathrm{e} 5$ & $8.944150 \mathrm{r} 4$ & Placenta abruption \\
\hline Image2 & $1.832 \mathrm{e} 4$ & 1614852030 & $2.978678 \mathrm{e} 6$ & $1.047565 \mathrm{e} 5$ & placenta accreta \\
\hline Image3 & $1.454 \mathrm{e} 4$ & 1434646325 & $1.911394 \mathrm{e} 5$ & $9.609855 \mathrm{e} 4$ & GDM complicating pregnancy \\
\hline Image4 & $1.455 \mathrm{e} 4$ & 1436691775 & $1.915322 \mathrm{e} 5$ & $9.670916 \mathrm{e} 4$ & Placental dy sfunction \\
\hline Image5 & $1.222 \mathrm{e} 4$ & 1077321331 & $1.055089 \mathrm{e} 5$ & $7.347292 \mathrm{e} 4$ & Normal placenta \\
\hline
\end{tabular}

\section{RESULTS}

As per the literature the placenta images were grouped based on the Grannum classification to assess the maturity of the placenta to assess the growth of the fetus. The proposed technique identifies the implications of diabetes mellitus on the growth of the placenta. This classification would help the sonologists to identify the abnormalities in the ultrasound and can prevent stillbirth or fetal demise.
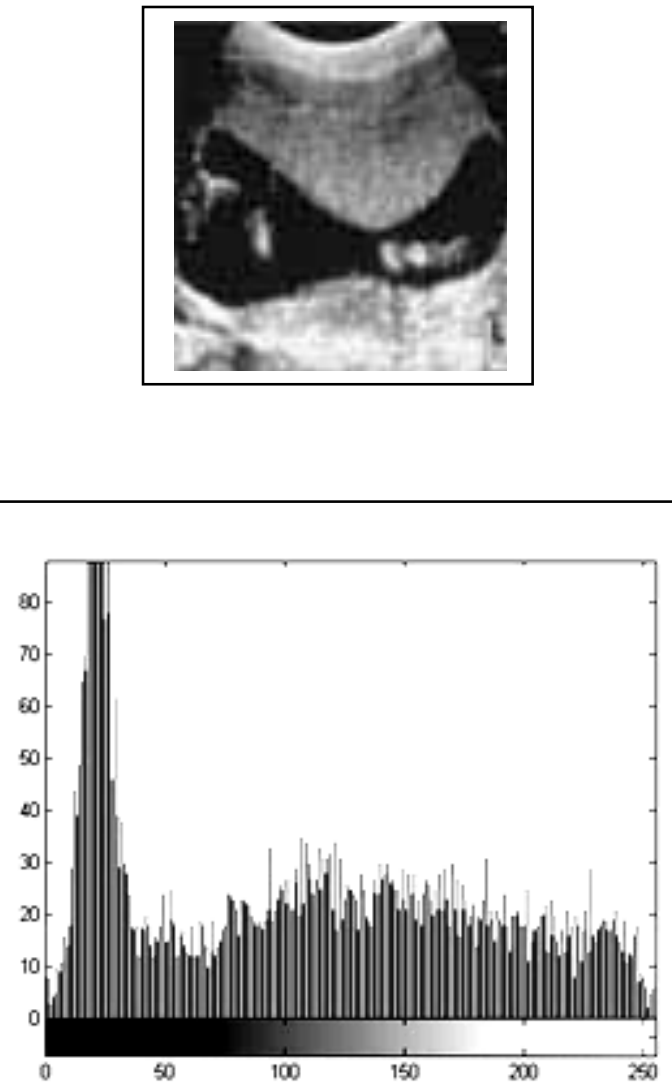

Fig2. Ultrasound Image of Normal Placenta and its histogram obtained using Matlab
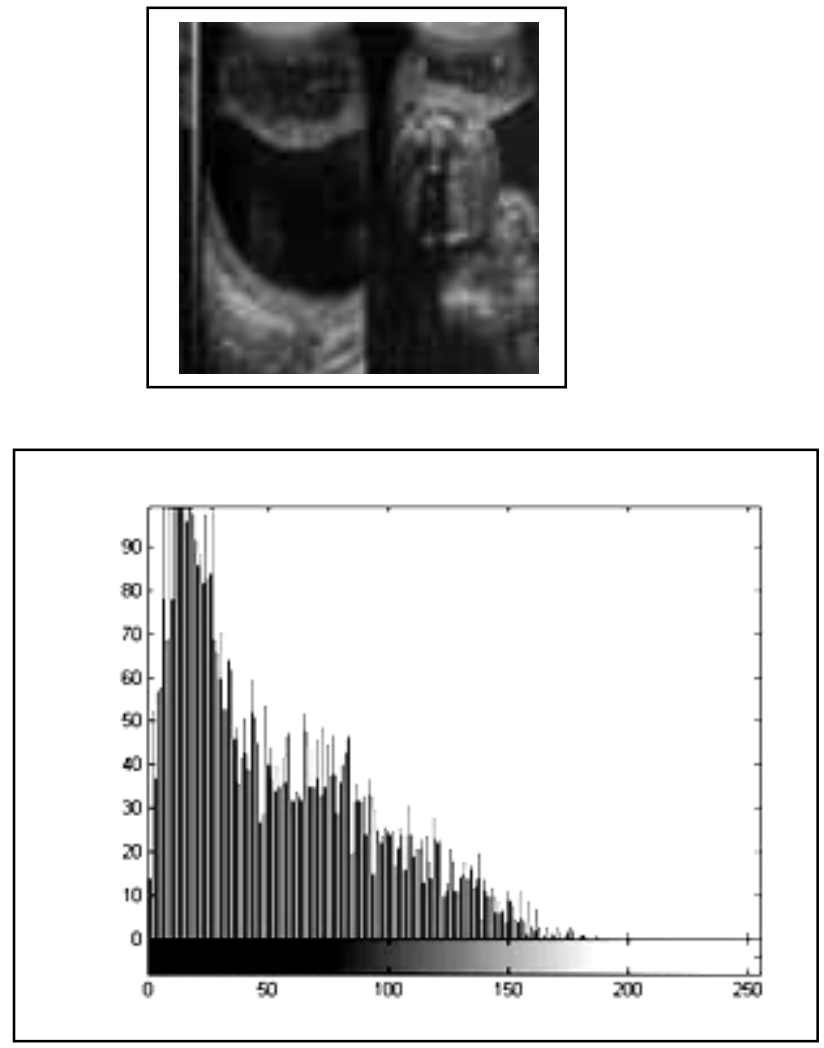

Fig2. Ultrasound Image of Abnormal Normal Placenta and its histogram obtained using Matlab

The histogram plot represent the frequency of pixel brightness against the number of gray levels as can be sen from the Matlab simulated plots

\section{ACKNOWLEDGMENTS}

Our thanks to Mr. Ramakrishnan, III year, Department of Computer Applications, Velammal Engineering College for helping us in the implementation. 


\section{REFERENCES}

[1] Diabetes news at: Www.diabetesnews.in/2006/12/diabetesstatistics.html

[2] New treatment for diabetes at: Www.new-treatment-fordiabetes-types.com/facts-about- diabetes-3.html

[3] Rasmussen S, Irgens LM, Dalaker K. A history of placental dysfunction and risk of placental abruption. http ://www.ncbi.nlm.nih.gov/pubmed/9987782

[4] Concise Medical Dictionary, Oxford University Press, Market House Books Ltd,1998

[5] The Times of India, Chennai, Tuesday, August,04, 2009, Published in American Journal of Perinatology, $3^{\text {rd }}$ August, 2009

[6] Placental Dysfunction-Placental Insufficiency. http://www.medicineonline.com/articles/P/2/PlacentalDysfunction/Placental-Insufficiency.html

[7] Meghana Toal, Vandana Chaddha, Rory Windrim, John Kingdom, “ Ultrasound Detection of Placental Insufficiency in Women with Elevated Second Trimester Serum Alpha Fetoprotein or Human Chorionic Gonadotropin", OBSTETRICS, March 2008

[8] Marek Pietryga, Jacek Brązert, Ewa Wender-O ègowska, Romuald Biczysko, Mariusz Dubiel, Saemundur Gudmundsson, "Abnormal Uterine Doppler Is Related to Vasculopathy in Pregestational Diabetes Mellitus", American Heart Association, Inc., 2005.

[9] P.A. Linares, P.J. Mc Cullagh, N.D. Black , J.Dornan, "Characterization of ultrasonic images of the placenta based on textural features", IEEE Conf. On Information Technology Application in Biomedicine, UK, 2003
[10] K.Thangavel, M.Karnan, A. Pethalakshmi, "Performance Analysis of Rough Reduct Algorithms in Mammogram", International Journal on Graphics, Vision and Image Processing, 2005

[11] P.A. T. Grannum, R.L. Nerkowitz and J.C. Honnins, "The Ultrasonic changes in the maturing placenta and their relation to fetal pulmonic maturity", American Journal of Obstetrics and Gy necology, 1979

[12] Byng.J.N, Boyd N.F. Fisheld, et al,"The Qualitative analysis of mammography densities", Physics in Medicine and Biology, 1994

[13] P.A. Linares, P.J. , N.D McCullagh, J Dornan, “ Feature Selectin for the characteriztion of ultrasonic images of the placenta using texture classification", IEEE International Symposium on Biomedical Imaging, 2004

[14] Prof. S.K. shah, V. Gandhi, " Image Classificatin Based on Textural Features using Artifical Neural Network(ANN), IE(I) Journal-ET, 2004

[15] M.J. Swain, D.H. Ballard,'Indexing via Color Histograms", Unversity of Rochester, Rochester

[16] Y.Rui, T.S. Huang,"Image Retrieval: Current Techniques, Promising Directions and Open Issues", Illinois, 1999

[17] Y.Rui, A.C. She, T.S. Huang, "Modified Fourier Descriptor for Shape Representation", University of Illinois

[18] R. Swiniarski, L.Hargis, "Rough sets as a front end of neuralnetworks texture classifiers", Neurocomputing, 2001

[19] Robert M. Haralick, K.Shanmugam, Its'Hak Dinstein, "Textural Features for Image Classification", IEEE Transactions on Systems, M an and Cybernetics, 1973 